\title{
Integrating optical sensing into lab-on-a-chip systems
}

\author{
Roberto Osellame, Rebeca Martínez Vázquez, \\ Roberta Ramponi, and Giulio Cerullo
}

A combination of waveguides and microfluidics is easily achieved through femtosecond-laser micromachining.

A lab-on-a-chip (LOC) device implements the functionalities of a biological laboratory on a single substrate through a network of microfluidic channels, reservoirs, valves, pumps, and microsensors. Its advantages are high sensitivity, analysis speed, low sample consumption, and measurement automation and standardization, thus promising significant advances in both basic research and clinical applications as a low-cost diagnostic tool. The greatest ongoing challenge is development of integrated onchip optical detection. ${ }^{1}$ Although optical-detection devices can achieve the low detection limits required, this is typically implemented using external systems that do not offer the robustness, stability, or operator independence of fully integrated approaches.

Traditional LOC and optical-waveguide fabrication techniques require clean-room facilities, photolithography, and complex multilayer processing to generate 3D structures. A novel approach, based on femtosecond-laser micromachining, has emerged as a powerful tool for both microfluidic-channel production and direct fabrication of optical waveguides and photonic circuits in transparent glass substrates. ${ }^{2}$ The technique's 3D capabilities enable easy integration of the waveguides with the microfluidic circuits, which may be either fabricated by the same femtosecond laser or designed to expand a commercial LOC.

Femtosecond lasers emit ultrashort light pulses $\left(\approx 10^{-13} \mathrm{~s}\right)$ that generate very high peak intensities when focused by a microscope objective lens. The resulting interaction of the laser pulse with the glass becomes strongly nonlinear, and a very selective absorption of energy occurs in a small volume around the focus. This induces a permanent modification in the material that, in accordance with the irradiation parameters of the light source and substrate, can result in either a positive refractive-index change or creation of nanocracks. By moving the laser focus inside the glass we can produce, respectively, 3D waveguides or

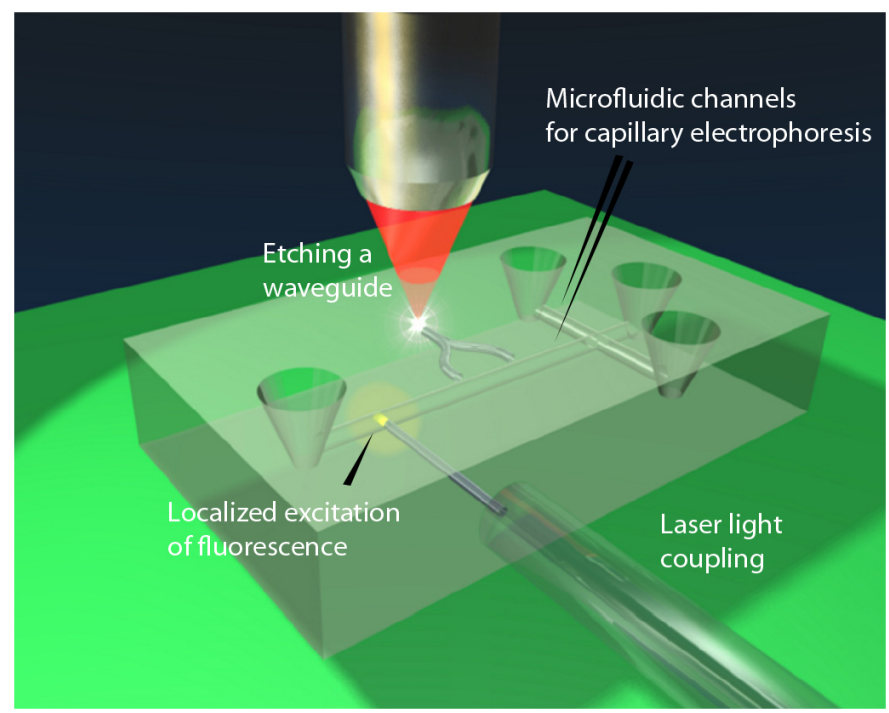

Figure 1. Femtosecond-laser direct writing is used to add optical waveguides to a lab-on-a-chip device designed for capillary electrophoresis. The optical waveguides, coupled to optical fibers, excite fluorescence in a highly localized region in the microchannel and guide emissions to a detector, resulting in on-chip sensing (not shown).

microchannels by selectively removing the damaged line in a subsequent chemical etching process. ${ }^{3}$ Thus, the technique does not require multiple layers to generate the $3 \mathrm{D}$ structure, and hence eliminates the need for clean-room environments.

Several optofluidic applications can be addressed with this technology, and may benefit from having both the fluidic and optical components fabricated by the same machining tool. The technique's ease of use even allows us to produce the optical waveguides in a commercial microfluidic chip, where the microchannel network has already been generated and optimized. We can thus add on-chip optical detection without affecting the LOC's layout and manufacturing, for example to integrate optical waveguides into a commercial LOC for capillary electrophoresis (see Figure 1). We fabricate high-quality optical

Continued on next page 
waveguides intersecting the separation channel, which we use to optically address the biomolecules flowing through it with high spatial selectivity. ${ }^{4}$ We can also fabricate waveguide splitters to accommodate multipoint sensing. In Figure 1, fluorescence from the optically excited volume is collected at a $90^{\circ}$ angle by an optical fiber (not shown) glued to the bottom of the chip. This results in a highly compact, portable device. We used the fluorescent dye Rhodamine 6G to test the design. Microscope images show highly localized yellow fluorescence at the waveguide $(\approx 10 \mu \mathrm{m}$, the waveguide's diameter) when the channel is filled with a dye solution, which is beneficial for high-resolution bioassays: see Figure 2(a). The device's functioning is validated by detecting different concentrations of dye-labelled oligonucleotides undergoing capillary electrophoresis on the chip. Electropherograms corresponding to concentrations of 1 and 10nM of a Cy3-labelled 23-mer oligonucleotide demonstrate very good sensitivity: see Figure2(b). ${ }^{5}$

In summary, femtosecond-laser micromachining of glass substrates is a very promising technique for fabricating optofluidic devices. In particular, on-chip detection using femtosecondlaser-written waveguides can overcome the present limitation of microfluidic devices coupled to macroscopic bulk opticaldetection systems. Compared with traditional microfabrication techniques, the approach provides some striking advantages. It is maskless, so that there is no need for clean rooms, while it also has unique 3D capabilities and provides both photonic and fluidic components. Our future work will focus on fabrication of interferometers in LOCs to provide label-free sensing and development of monolithic devices for cell sorting and optical analysis.

This work was funded by the European Commission's 6th Framework Programme-Specific Targeted Research Projects (STREP), contract IST-2005-034562: Hybrid Integrated Biophotonic Sensors Created by Ultrafast laser Systems (HIBISCUS).

\section{Author Information}

\section{Roberto Osellame and Rebeca Martínez Vázquez}

Istituto di Fotonica e Nanotecnologie - Consiglio Nazionale delle Ricerche (IFN-CNR)

Milano, Italy

Roberto Osellame is a research associate. He is also a contract professor at the Politecnico di Milano, Italy. His scientific interests include nonlinear and integrated optics, optical communications, femtosecond-laser micromachining, and LOC technologies. He is the coordinator of the European Commission project
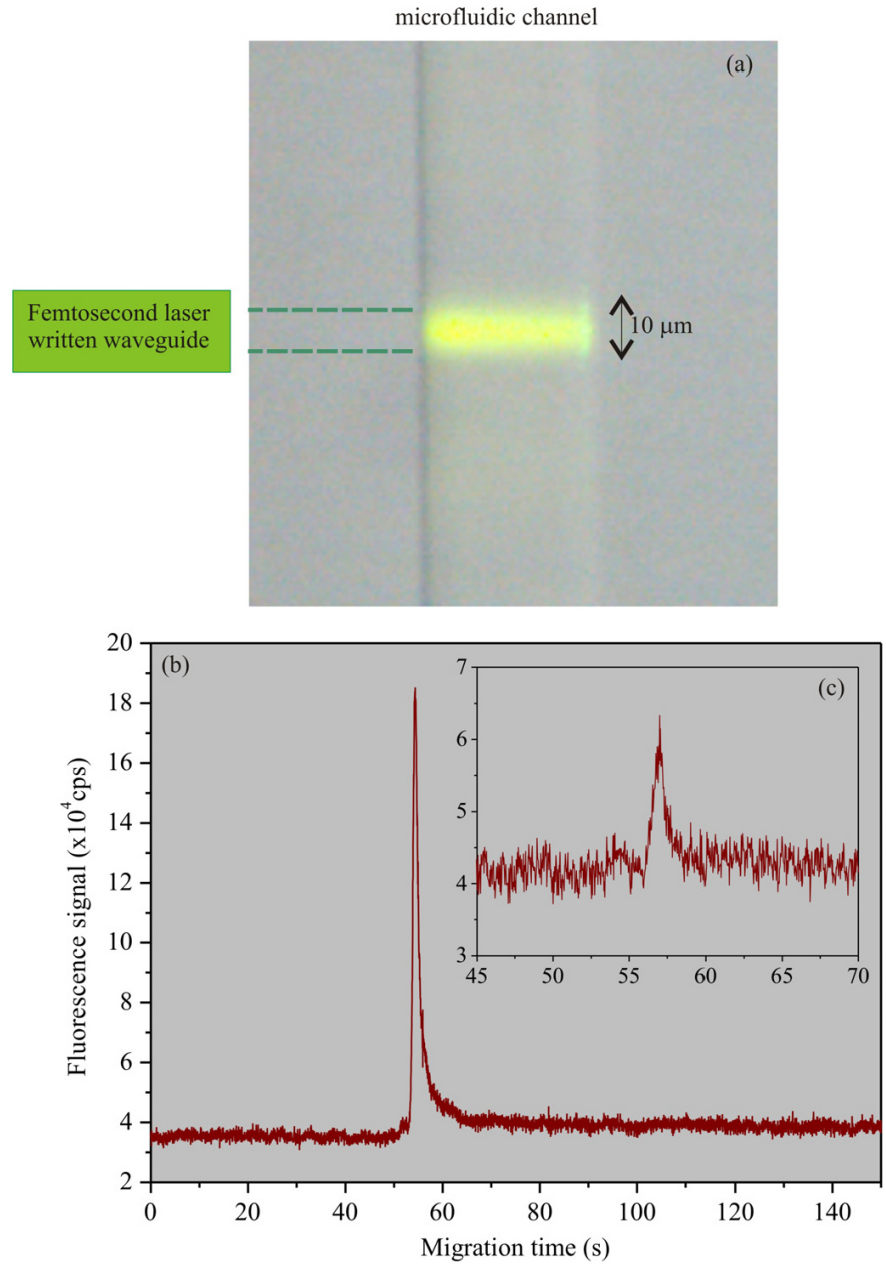

Figure 2. Microscopic image of the fluorescence signal excited in the microchannel by the optical waveguide. (a) Fluorescence induced in the the optical waveguide. (b) and (c) Electropherograms, corresponding to 10 and $1 \mathrm{nM}$ of dye-labelled oligonucleotides, respectively, measured at the end of the separation channel by the on-chip detection system.

microFLUID, which focuses on femtosecond-laser LOC microfabrication in polymers.

Rebeca Martínez Vázquez studied physics at the Autónoma University of Madrid, Spain, where she also obtained her $\mathrm{PhD}$. Since 2005 she has been a contract researcher at the IFN-CNR. Her research interests include crystal growth, solidstate physics, material science, integrated optics, and-more recently-biophotonics. 


\section{Roberta Ramponi and Giulio Cerullo}

Dipartimento di Fisica

Politecnico di Milano

Milano, Italy

Roberta Ramponi is a full professor of both physics and microand nano-optics. Her research activities include integrated and nonlinear optics, and photonic devices for applications to telecommunications, and to biomedical and environmental sensing. She was president of the European Optical Society from 2006 to 2008 .

Giulio Cerullo is an associate professor of physics. His research interests concern the generation of few-optical-cycle light pulses, their application to time-resolved spectroscopy, and femtosecond-laser microstructuring of materials. $\mathrm{He}$ is the coordinator of the European Commission project HIBISCUS, set up around the integration of femtosecond-laser-written waveguides on LOC devices.
References

1. E. Verpoorte, Chip vision-optics for microchips, Lab Chip 3, pp. 42N-52N, 2003. doi:10.1039/b3007027a

2. R. Gattass and E. Mazur, Femtosecond laser micromachining in transparent materials, Nat. Photon. 2, pp. 219-225, 2008. doi:10.1038/nphoton.2008.47

3. R. Osellame, V. Maselli, R. Martínez Vázquez, R. Ramponi, and G. Cerullo, Integration of optical waveguides and microfluidic channels both fabricated by femtosecond laser irradiation, Appl. Phys. Lett. 90, p. 231118, 2007. doi:10.1063/1.2747194

4. R. Martínez Vázquez, R. Osellame, D. Nolli, C. Dongre, H. van den Vlekkert, R. Ramponi, M. Pollnau, and G. Cerullo, Integration of femtosecond laser written optical waveguides in a lab-on-chip, Lab Chip 9, pp. 91-96, 2009. doi:10.1039/b808360f

5. R. Martínez Vázquez, R. Osellame, M. Cretich, M. Chiari, C. Dongre, H. Hoekstra, M. Pollnau, H. van der Vlekkert, R. Ramponi, and G. Cerullo, Optical sensing in microfluidic lab-on-a-chip by femtosecond-laser-written waveguides, Anal. Bioanal. Chem. 393, pp. 1209-1216, 2009. doi:10.1007/s00216-008-2399-8 